\title{
Circle of Willis Configuration as a Determinant of Intracranial Dolichoectasia
}

\author{
Jose Gutierrez ${ }^{\mathrm{a}}$ Sally Sultan ${ }^{\mathrm{a}}$ Ahmet Bagcic Tatjana Rundek ${ }^{\mathrm{d}}$ Noam Alperin ${ }^{\mathrm{c}}$ \\ Mitchell S.V. Elkind ${ }^{a, b}$ Ralph L. Sacco ${ }^{d}$ Clinton B. Wright ${ }^{d}$ \\ ${ }^{a}$ Department of Neurology, Columbia University Medical Center, and bepartment of Epidemiology, Mailman School \\ of Public Health, Columbia University Medical Center, New York, N.Y., and Departments of ${ }^{\mathrm{c}}$ Radiology and ${ }^{\mathrm{d}}$ Neurology, \\ Evelyn F. McKnight Brain Institute, Leonard M. Miller School of Medicine, University of Miami, Miami, Fla., USA
}

\section{Key Words}

Dolichoectasia $\cdot$ Circle of Willis anatomy $\cdot$ Arterial diameters

\begin{abstract}
Background: Circle of Willis (COW) variants might influence arterial caliber in the brain. We hypothesized that these variants would be associated with the prevalence of intracranial dolichoectasia (DE). Methods: We examined COW variants and $D E$ in a sample of stroke-free participants $(n=436)$ undergoing magnetic resonance angiography (MRA) as part of a population-based study. Large intracranial arterial diameters were obtained when available; if not, the artery was defined as hypoplastic or absent according to its visibility on MRA. Subscores for the anterior and the posterior circulations were created. DE was defined as arterial diameters $\geq 2$ SD above the population mean for that artery, adjusting for intracranial volume. Generalized linear models with a Poisson distribution were used to evaluate predictors of both absent and hypoplastic vessels, and logistic regression was used to assess the odds ratio (OR) and $95 \%$ confidence interval $(95 \% \mathrm{Cl})$ of DE depending on COW variants. Results: Only $44 \%$ of the sample had all 14 arteries present, 32\% lacked 1 artery, $18 \%$ lacked 2 and $6 \%$ lacked 3 or more. DE of at
\end{abstract}

least 1 artery was not associated with the total number of hypoplastic or absent arteries, but DE in a posterior circulation artery was weakly associated with the number of absent arteries in the posterior circulation ( $\beta$ coefficient $=0.36, p=$ $0.06)$. DE of at least 1 artery was more frequent in those with 1 or more absent arteries (OR 1.27, 95\% Cl 1.03-1.57). Posterior circulation $D E$ was more frequent in participants with at least 1 or more absent arteries at any location (OR 1.35, 95\% Cl 1.02-1.78). Participants with an incomplete posterior COW were more likely to have DE in the anterior circulation (OR 1.52, 95\% Cl 1.01-2.33). Having an absent left anterior cerebral artery (ACA) A1 segment was associated with right ACA DE (OR 34.1, 95\% Cl 3.16-368.2); an absent right ACA was associated with left ACA DE (OR 14.1, 95\% Cl 1.69118.28). Absence of 1 (OR 1.9, 95\% Cl 1.1-3.4) or 2 (OR 3.0, $95 \% \mathrm{Cl} 1.4-6.6)$ of the 2 arteries connecting the anterior to the posterior circulation was associated with basilar artery DE. Conclusion: The COW is a pleomorphic structure that allows collateral flow to compensate for an insufficient or absent arterial component at the base of the skull. By presumed flow diversion, arteries might undergo outward remodeling. Whether this compensatory arterial dilatation is beneficial or not remains unknown.

(c) 2013 S. Karger AG, Basel

\section{KARGER}

(C) 2013 S. Karger AG, Basel

1015-9770/13/0366-0446\$38.00/0

E-Mail karger@karger.com

www.karger.com/ced
Jose Gutierrez, MD, MPH

Department of Neurology, Columbia University Medical Center

$710 \mathrm{~W} 168$ th Street, 6th floor

New York, NY 10032 (USA)

E-Mailjg3233@cumc.columbia.edu 


\section{Introduction}

Intracranial dolichoectasia (DE) is an arterial disease that manifests with larger-than-expected luminal diameters and tortuosity $[1,2]$. DE has been associated with an increased risk of both ischemic and hemorrhagic cerebrovascular events, and with compressive symptoms such as cranial neuropathies and hydrocephalus [3-8]. Progressive DE may increase the risk of recurrent stroke and mortality $[9,10]$. The mechanism leading to the dilatation or outward vascular remodeling of dolichoectatic arteries is unknown. Animal and human models suggest that increased flow induces physiological outward arterial remodeling, and DE is one pathological phenotype of this response [11-15]. The capacity of each artery in the Circle of Willis (COW) determines the flow dynamics in the large intracranial arteries. Because the configuration of the COW determines the flow distribution at the base of the skull and, consequently, the caliber of the intracranial large arteries, it is plausible that flow-induced arterial remodeling and DE prevalence might be affected by these various configurations.

The aim of this analysis is to determine the degree to which anatomic variants in the COW are associated with intracranial arterial calibers and DE. In addition, we explored whether any associations between caliber and collaterals might be affected by cardiovascular risk factors.

\section{Methods}

The Northern Manhattan Study (NOMAS) identified stroke-free participants using random digit dialing with dual-frame sampling to identify published and unpublished telephone numbers [16]. In 2003, an institutional review board-approved magnetic resonance imaging (MRI) substudy was nested into the ongoing NOMAS study. Surviving stroke-free members older than 55 years were invited to participate, and those who consented were screened for eligibility for MRI scanning. Imaging was performed on a 1.5-tesla MRI system (Philips Medical Systems) at the Hatch Research Center following a standardized protocol. We used 3-dimensional (3D) timeof-flight (TOF) magnetic resonance angiography (MRA) with the following parameters: a field of view of $15 \mathrm{~cm}, 1 \mathrm{~mm}$ effective slice thickness, the acquisition matrix was interpolated to $256 \times 228$ matrix, a flip angle of $25^{\circ}$, repetition time/echo time $20 / 2.7 \mathrm{~ms}$. There were 741 subjects with MRA data available for semi-automated arterial diameter measurements, with 35 excluded from this analysis because of motion artifact and 270 excluded due to incomplete data on the intracranial vertebral arteries (VAs), leaving 436 subjects with the complete information on the COW required for this analysis.

Sociodemographic data, including age, sex, race and ethnicity, were obtained by self-report. Information on the presence of hypertension (HTN), diabetes mellitus (DM), hypercholesterolemia, smoking and prior cardiac disease was collected as described elsewhere [16].

Circle of Willis and Dolichoectasia
Morphological parameters of all available intracranial arteries, including arterial diameter, were obtained using a semi-automated $3 \mathrm{D}$ vascular analysis tool that incorporates automated tracking of the arterial centerline [17]. One of three possible descriptions was recorded for each intracranial artery: visible in the $3 \mathrm{D}$ format reconstruction, not visible in the $3 \mathrm{D}$ format due to a very small size but visualized in the MRA axial source images (i.e. hypoplastic), or not visible in either the 3D format or the MRA axial images (i.e. absent artery). The threshold for the $3 \mathrm{D}$ reconstruction was systematically set to the point of maximal pixel saturation of the arteries that avoided parenchymal pixel visualization (intraclass correlation coefficient for threshold $=0.998$ ).

The anterior part of the COW included the internal carotid arteries (ICAs), the middle cerebral arteries (MCAs), the anterior cerebral arteries (ACAs) and the anterior communicating artery (Acomm). The Acomm was described as 'present' or 'absent'. It was not measured due to the inability to isolate it from the ACA with the measuring tool. The posterior part of the COW included the posterior cerebral arteries (PCAs), the posterior communicating arteries (Pcomms) and the basilar artery (BA). Although not part of the COW, we included the VAs as part of the posterior aspect of the COW since they may be a contributing determinant of BA size. The total number of arteries per subject that were hypoplastic was used to create a 'hypoplastic artery' score, and the total number of absent arteries was used to create an 'absent artery' score. DE by arterial diameters was defined as head size-adjusted arterial diameter $\geq 2 \mathrm{SD}$ above the mean for this population, as described before [18]. All scans were assessed by one reader (J.G.). Measures of reproducibility were collected for the arterial diameters and the categorization of the state of the arteries and were good to excellent [18]. In the intra-reader agreement analysis, the characterization of arteries as absent, hypoplastic and measured all had $\kappa$ values $>0.75$.

\section{Statistical Analysis}

Arterial diameters across arteries were compared using Student's t test if normally distributed as determined by the ShapiroWilk test. To evaluate the association of DE with specific variants in the anterior or posterior circulation, univariate and multivariable logistic regressions were used to obtain odds ratios (OR) and $95 \%$ confidence intervals ( $95 \% \mathrm{CI})$. To evaluate for a differential effect between the anterior versus posterior circulations, the scores corresponding to the ICAs, MCAs, ACAs and Acomms were considered to represent the anterior circulation and those of the PCAs, VAs, Pcomms and the BA were considered to represent the posterior circulation. To further elucidate the relationship between the COW and DE, we analyzed the integrity of the COW by categorizing it as incomplete posterior COW (defined as 3 or more hypoplastic arteries and/or any absent artery) and/or incomplete anterior COW (defined as 3 or more hypoplastic arteries and/or any absent artery). A second analysis was focused on the association between the number of hypoplastic and absent arteries and DE, as well as cardiovascular risk factors, using the hypoplastic and absent scores as dependent variables. The hypoplastic and absent scores had means almost equal to their variances. For these scores, a generalized linear model with Poisson distribution was used to evaluate predictors. A p value $<0.05$ was considered statistically significant for associations and a $p$ value $<0.10$ for interactions. The statistical software used for the analysis was IBM SPSS Statistics 20 (Release 20.0.0, IBM, 2011). 
Fig. 1. The five most common COW variants of the anterior and posterior circulation in the NOMAS.

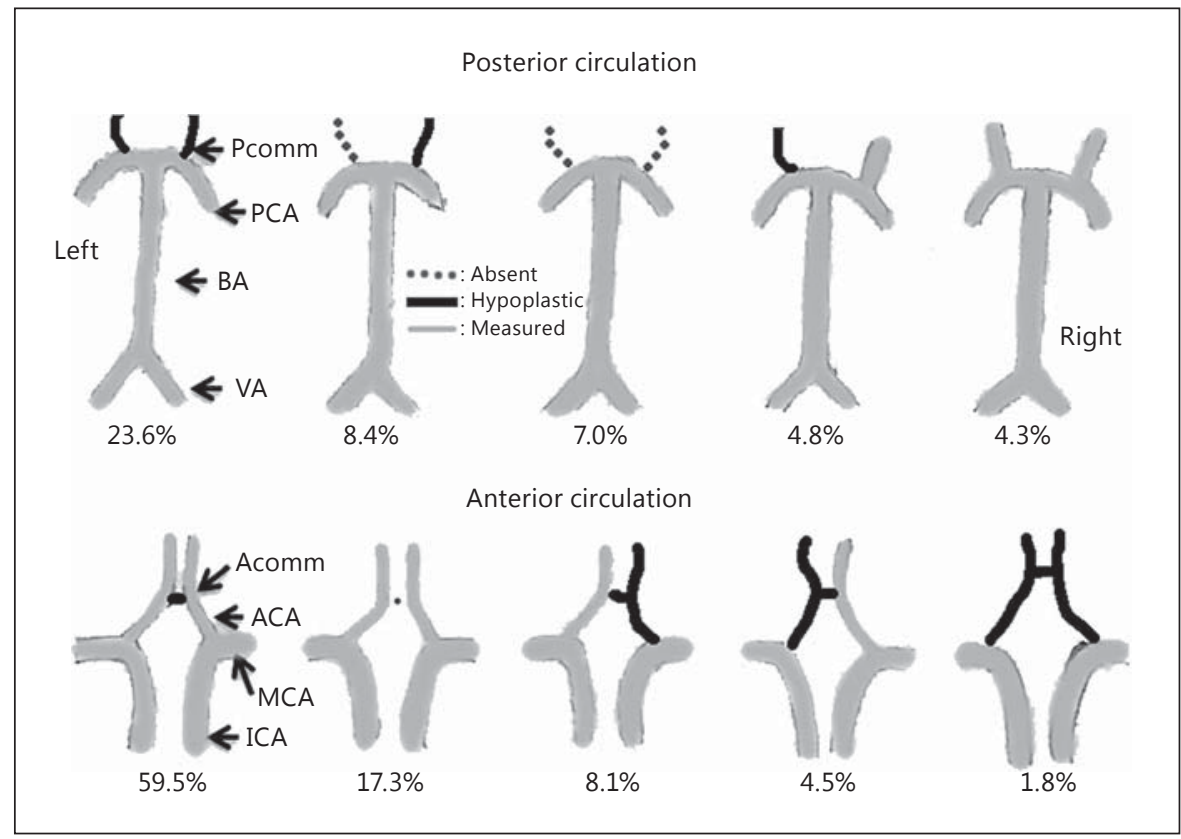

Table 1. Characteristics of the sample studied

\begin{tabular}{ll}
\hline Mean age \pm SD, years & $72 \pm 8$ \\
Female sex, \% & 35 \\
Race/ethnicity, \% & \\
$\quad$ Non-Hispanic white & 15 \\
$\quad$ Hispanic & 66 \\
$\quad$ Black & 19 \\
Mean body mass index \pm SD & $28 \pm 5$ \\
HTN, \% & 71 \\
DM, \% & 19 \\
Hypercholesterolemia, \% & 56 \\
Current smoking, \% & 16 \\
Prior cardiac disease, \% & 16 \\
Absent artery score, \% & \\
$\quad$ Median & 1 \\
IQR & 1 \\
Range & $0-3$ \\
Hypoplastic artery score & \\
Median & 2 \\
IQR & 1 \\
Range & $0-7$ \\
Any DE, \% & 19 \\
Anterior circulation & 12 \\
Posterior circulation & 10 \\
\hline
\end{tabular}

$\mathrm{SD}=$ Standard deviation; $\mathrm{IQR}=$ interquartile range

\section{Results}

\section{Description of the Cohort}

There were 436 subjects for whom complete information on all 14 intracranial arteries could be analyzed. Compared to the complete NOMAS cohort, the subjects included in the COW analysis were less likely to have a history of heart disease and to be Hispanic, but were older (table 1).

\section{COW Variants in the Cohort}

We identified 22 types of variants of the anterior portion of the COW and 75 variants of the posterior portion (the most common types are shown in fig. 1). Only $44 \%$ of the sample had all 14 arteries present; $32.2 \%$ lacked 1 artery, $17.7 \%$ lacked 2 and $6.1 \%$ lacked 3 or more. In general, the posterior circulation arterial configuration was more pleomorphic than the anterior circulation. The posterior circulation was 2 times more likely to lack at least 1 artery than the anterior circulation (OR 2.13, 95\% CI $1.44-3.31)$. The majority of the subjects had at least 1 hypoplastic artery $(84.6 \%)$ with $10 \%$ having 4 or more hypoplastic arteries. The posterior circulation was also more likely to have at least 1 hypoplastic artery compared to the anterior circulation (OR 1.88, 95\% CI 1.01-3.49). The Pcomms were the most commonly absent or hypoplastic arteries, and $10.9 \%$ of the sample did not have any artery connecting the posterior and anterior circulations [i.e., 
Table 2. Associations between COW variants and intracranial $\mathrm{DE}^{\mathrm{a}}$

\begin{tabular}{llll}
\hline & Any DE & Anterior circulation DE & Posterior circulation DE \\
\hline Total absent score $^{\mathrm{b}}$ & $1.27(1.03-1.57)$ & $1.13(0.83-1.55)$ & $1.35(1.02-1.78)$ \\
$\quad$ Anterior circulation $_{\text {Posterior circulation }}$ & $1.22(0.72-2.01)$ & $1.30(0.71-2.40)$ & $0.89(0.44-1.81)$ \\
Total hypoplastic score $^{\mathrm{b}}$ & $1.27(0.91-1.79)$ & $1.10(0.73-1.64)$ & $1.54(1.01-2.37)$ \\
$\quad$ Anterior circulation & $0.96(0.78-1.17)$ & $0.94(0.74-1.19)$ & $0.98(0.76-1.26)$ \\
$\quad$ Posterior circulation & $1.06(0.66-1.70)$ & $0.99(0.66-1.54)$ & $1.13(0.80-1.61)$ \\
Incomplete anterior COW & $0.88(0.68-1.13)$ & $0.91(0.68-1.22)$ & $0.94(0.69-1.30)$ \\
Incomplete posterior COW & $1.28(0.75-2.18)$ & $1.41(0.77-2.62)$ & $0.88(0.42-1.81)$ \\
\hline
\end{tabular}

a All estimates are adjusted for age, sex, race/ethnicity, HTN, DM, hypercholesterolemia and current smoking. ${ }^{b}$ The total absent and hypoplastic scores represent the sum of all absent or hypoplastic large arteries in the brain; posterior and anterior represent the partial sum corresponding to the arteries in either the anterior or the posterior circulation.

anterior-posterior (AP) arteries in the form of fetal PCA (fPCA) or Pcomm were absent]. The PCA had a fetal origin in $13.4 \%$ of the sample $(6.8 \%$ on the right, $6.1 \%$ on the left and $0.5 \%$ both). A quarter of the subjects lacked a visible Acomm (24.0\%).

\section{Variants of the COW and DE}

$\mathrm{DE}$ of at least 1 artery was more frequent in those with 1 or more absent arteries (OR 1.27, 95\% CI 1.03-1.57; table 2). Analyzing anterior and posterior circulation DE separately, we found an association between the total number of absent arteries (OR 1.35, 95\% CI 1.02-1.78 per absent artery) and the number of absent arteries in the posterior circulation $(1.54,95 \%$ CI $1.01-2.37$ per absent artery) with posterior circulation DE but not with anterior circulation DE (table 2). Hypoplastic arteries alone, expressed as total score or divided into anterior or posterior circulation, were not associated with total DE or DE in the anterior or posterior circulation. An incomplete posterior aspect of the COW was associated with anterior circulation DE (OR 1.47, 95\% 1.00-2.20). Adjusting for demographics and cardiovascular risk factors strengthened the association (OR for anterior circulation DE in the presence of incomplete posterior DE 1.53, 95\% CI 1.02-2.29).

We further found that participants with an absent A1 segment were more likely to have contralateral ACA DE. Absence of the left Al segment was associated with a greater odds of having right $\mathrm{A} 1 \mathrm{DE}$ when an Acomm was present (OR 34.1, 95\% CI 3.16-368.2), but this association was not seen when the Acomm was absent (OR 1.15, 95\% CI 0.08-15.81). Similarly, absence of the right A1 segment was associated with left ACA DE (OR 14.1, 95\%
CI 1.69-118.28; fig. 2b). Having an absent left ACA was associated with greater diameters in the right ACA ( $p=$ $0.10)$ and the right MCA ( $<<0.001$; fig. $2 b)$.

We found a relationship between the diameters of the $\mathrm{BA}$ and the ICA depending on the COW configuration connecting both arterial trees. For example, the ICA diameter was larger in the presence of ipsilateral fPCA or a dominant Pcomm defined as Pcomm diameter greater than ipsilateral PCA diameter ( $4.6 \mathrm{vs} .4 .3 \mathrm{~mm}$ for the right COW and 4.6 vs. $4.3 \mathrm{~mm}$ for the left COW, p $<0.001$ for both comparisons). In an inverse fashion, the BA was smaller in individuals with both AP arteries (either Pcomm or fPCA) present compared to those without them (BA mean diameter 2.2 vs. $4.1 \mathrm{~mm}, \mathrm{p}<0.05$; fig. 3). BA diameter was on average $0.20 \mathrm{~mm}$ smaller for every 1 -mm increment in the average diameters of the AP arteries. These changes were not exclusive to the BA, but involved its parent vessels: the VA diameters were $0.16 \mathrm{~mm}$ smaller per $1-\mathrm{mm}$ increment in the average AP arterial diameters. Because DE was defined by diameters and because diameters changed depending on the COW configuration, DE was strongly associated with COW variants. For example, in an adjusted model including vascular risk factors, the odds of having BA DE followed an inverse dose-effect relationship depending on the number of AP arteries present (fig. 2a). When only 1 AP artery was present, compared with $2 \mathrm{AP}$ arteries being present, DE was common (OR 1.88, 95\% CI 1.05-3.37). The effect was greater when neither AP artery was present (OR 3.03, 95\% CI 1.39-6.58). We found that the degree of lateral deviation of the BA from its midline position relative to the clivus was positively associated with the degree of 


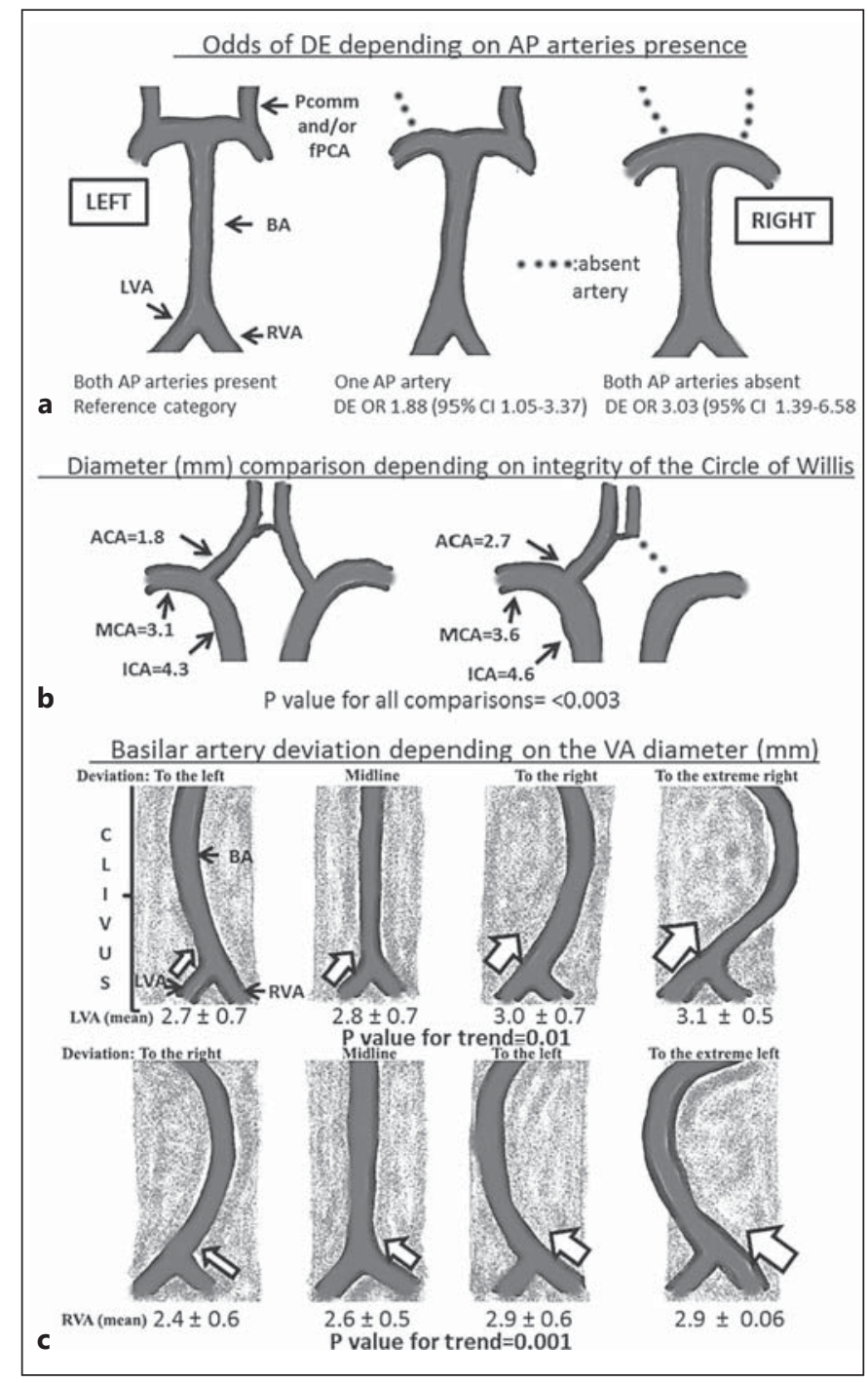

Fig. 2. Supporting evidence of flow-induced intracranial arterial remodeling. a The odds of BA DE are increased in a dose-effect fashion with the absence of 1 or 2 antero-posterior communicating arteries (either in the form of PPCA or Pcomm). $\mathbf{b}$ In the absence of the first segment of the ACA (A1), the contralateral diameters are increased compared to when the Al segment is present. $\mathbf{c}$ The degree of lateral deviation of the BA from its midline position relative to the clivus was positively associated with the degree of contralateral VA dominance.

contralateral VA dominance (as inferred from the difference in diameters between the two VAs; fig. 2c).

\section{COW Variants and Cardiovascular Risk Factors}

The number of absent arteries was not associated with any of the demographic or clinical variables included in the analysis. Both HTN and DM trended toward an as-
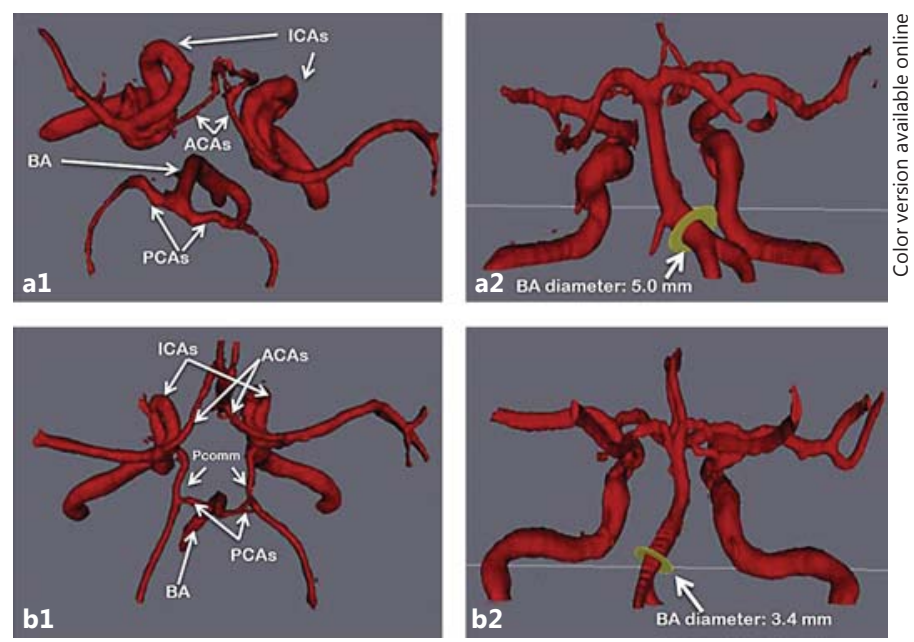

Fig. 3. BA DE and COW variants. Example of the BA diameter influenced by the status of antero-posterior arteries, in the form of Pcomm or fPCA. Top row case: a1 Note the lack of AP arteries in the top-down view of the COW. a2 Note the lateral deviation of the $\mathrm{BA}$ and its dilated origin measuring $5 \mathrm{~mm}$. Bottom row case: b1 Complete COW as demonstrated by presence of both Pcomm and both PCA. b2 The BA diameter is small; its pathway is less anfractuous than that of the top row case.

sociation with absence of arteries in the anterior circulation, but not in the posterior circulation (table 2). In multivariable analysis, no statistically significant association was found. However, we found a statistical interaction for participants with both HTN and DM $(\mathrm{p}=0.06)$ as well as Hispanics with HTN $(p=0.06)$ for a greater frequency of absent arteries in the anterior circulation. These interactions were not found for the posterior circulation.

Age (in years) and prior cardiac disease were positively associated with the total number of hypoplastic arteries, while Hispanics had fewer hypoplastic arteries in univariate analysis (table 3 ). Only age remained significant in the multivariable analysis $(\beta$ coefficient $=0.013, p=0.01)$. In the same adjusted model, age $(\beta$ coefficient $=0.05, \mathrm{p}<$ $0.001)$ and prior cardiac disease ( $\beta$ coefficient $=0.69, \mathrm{p}=$ 0.002 ) were the only significant predictors of the number of hypoplastic arteries in the anterior circulation; this association was not present for the posterior circulation.

\section{Discussion}

The configuration of the COW determines the distribution of blood at the base of the brain [19]. With the cross-sectional nature of our results in mind, our data 
Table 3. Unstandardized $\beta$ coefficients for unadjusted linear regression analysis

\begin{tabular}{|c|c|c|c|c|c|c|}
\hline & \multicolumn{3}{|c|}{ Absent artery score ${ }^{\mathrm{a}}$} & \multicolumn{3}{|c|}{ Hypoplastic artery score ${ }^{\mathrm{a}}$} \\
\hline & total & anterior & posterior & total & anterior & posterior \\
\hline Age, years & -0.004 & 0.001 & -0.006 & $0.015^{* *}$ & $0.054^{* * *}$ & 0.006 \\
\hline Female sex & -0.087 & 0.038 & -0.144 & -0.090 & -0.161 & -0.073 \\
\hline \multicolumn{7}{|l|}{ White } \\
\hline Hispanic & -0.031 & 0.369 & -0.188 & $-0.195^{*}$ & $-0.442^{*}$ & -0.131 \\
\hline Black & 0.005 & 0.347 & -0.109 & -0.048 & 0.225 & -0.001 \\
\hline Body mass index & -0.009 & 0.010 & -0.017 & -0.001 & -0.023 & 0.004 \\
\hline HTN & 0.042 & 0.337 & -0.082 & 0.100 & -0.001 & 0.125 \\
\hline $\mathrm{DM}$ & 0.023 & 0.299 & -0.122 & -0.009 & 0.083 & -0.031 \\
\hline Hypercholesterolemia & -0.223 & -0.410 & -0.145 & -0.029 & -0.190 & 0.007 \\
\hline Smoking & 0.052 & -0.121 & 0.126 & -0.082 & -0.267 & -0.042 \\
\hline Prior cardiac disease & 0.037 & -0.024 & 0.065 & $0.201^{*}$ & $0.650^{* * *}$ & 0.074 \\
\hline
\end{tabular}

p values: ${ }^{*} 0.05-0.011 ; * * 0.01-0.001 ; * * *<0.001$

a The total absent and hypoplastic scores represent the sum of all absent or hypoplastic large arteries in the brain; posterior and anterior represent the partial sum corresponding to the arteries in either the anterior or the posterior circulation.

suggest that flow may alter arterial shape and diameter in the brain. We hypothesized that absent and, to a certain degree, hypoplastic arteries induce outward remodeling in contralateral arteries due to increased flow, as evidenced by greater arterial diameters, in those vessels. Our analysis does not include changes over time and, thus, we cannot exclude the possibility that the opposite sequence of events is true, i.e. that factors that induce dolichoectatic changes in some arteries might induce stenosis or occlusion in other arteries. Even if this is true, the contralateral arterial diameters suggest that flow redistribution occurs, and it is compelling to imagine that $\mathrm{DE}$ is a compensatory response to compromised blood flow in the presence of small or absent components of the COW collateral network, although the time in life when these changes occur remains unknown. Following this logic, the extent of compensation appears to be greater when an artery is absent than when it is hypoplastic. This suggests that hypoplastic arteries are still capable of conducting some flow with less diversion into another artery, although we found that the cumulative presence of hypoplastic arteries is also associated with $\mathrm{DE}$. An incomplete posterior portion of the COW was associated with anterior DE in our sample, suggesting diversion of flow through the anterior circulation. This is further supported by an inverse relationship between hypoplasia or absence of AP arteries and the arterial diameters of the posterior circulation. Compensation for low flow is also evident in the anterior circulation, where larger arterial diameters were seen opposite to the side of an absent or small ACA or MCA (fig. 2).

Our findings offer further support to preliminary evidence showing the COW as a highly heterogeneous structure that might be able to undergo dynamic adaptive remodeling $[20,21]$. Some authors have effectively described the linear relationship between brain parenchyma volumes and distal small artery resistance to ipsilateral arterial diameters [20]. Because arterial resistance in the more distal field is influenced by the metabolic demands of the brain, one can argue that changes in brain metabolic demand can have an impact in the large arteries' caliber in the COW, aimed at increasing the flow to that area [22]. Although DE has generally been thought to represent arterial pathology, our data suggest that DE might also represent compensatory luminal dilatation in response to compromised collateral flow or brain parenchymal needs. The lack of compensatory arterial dilatation might represent inadequate collaterals and could potentially be associated with higher stroke risk or worse stroke outcomes in the absence of other extracranial or leptomeningeal sources of collateral flow [23].

In our sample, a full and complete COW was present in a minority (44\%) of the sample. Hypoplastic and absent arteries were more common in the posterior COW, compared with its anterior portion. Compared with absent arteries in the anterior circulation, where acquired 
risk factors such as HTN and DM showed a trend for association, age and prior cardiac disease were significant risk factors for hypoplastic arteries in the anterior circulation. The observation that aging is associated with hypoplastic arteries was initially made by Padget [24], but it has not always been confirmed [25]. The relationship with age suggests that the number of hypoplastic arteries might be a consequence of aging, although the association with prior cardiac disease suggests that in some cases, a vascular cause might be present. Riggs and Rupp [26] demonstrated that in cadavers of individuals who died of unspecified neurological diseases, the prevalence of hypoplastic arteries was very high (79\%). Alpers et al. [25] found that in some cases, hypoplastic arteries have inward vascular remodeling consistent with atherosclerotic arterial occlusion. Unfortunately, the cross-sectional nature of our data limits our ability to speculate on cause and effect. The interaction of HTN with DM was the strongest correlate of absent arteries in the anterior circulation. It is important to acknowledge that these findings could either mean that some of these arteries were occluded or were so narrow due to stenosis or hypoplasia that flow-related enhancement was absent given our TOF MRA.

Contrary to our findings in the anterior circulation, it is surprising that the number of absent and/or hypoplastic arteries in the posterior circulation was not associated with any demographic and clinical characteristics in our sample, despite greater phenotypic variations. It is plausible that the embryology of the cerebrovascular system may help explain this finding. The main determinant of angiogenesis in the fetus is the rapidly growing demand for perfusion of the expanding brain parenchyma [2729]. The anterior circulation follows a more predictable configuration because it grows in an orderly fashion from the caudal portion of the embryonic cervical region to the growing frontal lobes and telencephalon. In contrast, the posterior circulation arises from gradual fusion of previous anastomoses in the neck that later become independent from the anterior circulation in the majority of cases when the occipital lobe begins to grow rapidly [30, 31].

The heterogeneous configuration of the COW was noted by Willis himself more than three centuries ago when he described its functionality [19]. Subsequent work demonstrated that the intact COW is found only in a minority. Alpers et al. [25] found that half of the studied subjects from an autopsy bank had a normal polygon, and a third had at least 1 filiform (or hypoplastic) artery. The most commonly absent or hypoplastic artery was the
Pcomm, similar to our findings [25]. The prevalence of a fetal origin to the PCA was reported in $14.6 \%$ of this autopsy sample. Our results are largely in accord with this estimate. It is important to note that in our group, the prevalence of hypoplastic arteries was much higher. There are a number of possible explanations. The NOMAS population is older, and we showed that age is strongly associated with the number of hypoplastic arteries. Additionally, the study methods to detect or define a hypoplastic artery were different (autopsy vs. MRA). In two other series that used MRA, one from the Netherlands and one from Australia, the prevalence of a complete COW was similar to ours $[32,33]$. The greater heterogeneity of the posterior circulation compared to the anterior circulation has also been documented in these series.

Our results should be framed in the context of various limitations. Sequential events in vascular remodeling are presumed, but our findings are cross-sectional and, thus, our inferences about remodeling are hypothetical. The precision of $3 \mathrm{D}$ TOF MRA is limited when there is slow flow in a given vessel, since it underestimates the diameters as compared to conventional angiogram, adding on error to our measurements [34]. Arteries categorized by us as absent may only have been hypoplastic, although the risk of misclassification with MRA does not exceed 20\% for the Pcomm and 10\% for the Acomm with MRA compared to conventional angiogram [35]. The NOMAS cohort was stroke free at enrolment, so the findings in this study might not be generalizable to people with stroke, in whom collateral vessels might have changed in reaction to the physiopathology of the ischemic event. The strengths of our analysis are the good reproducibility of measurements as well as the standardized methods of defining DE [18]. Arterial diameters derived from crosssectional areas also allowed us to make inferences about the underlying blood flow in corresponding vessels [17, $36,37]$.

In summary, the COW is a pleomorphic structure that allows collateral flow to compensate for an insufficient or absent arterial component at the base of the skull. We found that the posterior circulation is more pleomorphic compared to the anterior circulation, but pleomorphism in the anterior circulation is related to either HTN or DM, in the case of an absent artery, or to age in the case of a hypoplastic artery. By presumed flow diversion, arteries might undergo dilatatory changes. Whether this compensatory arterial dilatation is beneficial or not remains unknown. 


\section{References}

1 Smoker WR, Corbett JJ, Gentry LR, Keyes WD, Price MJ, McKusker S: High-resolution computed tomography of the basilar artery. 2. Vertebrobasilar dolichoectasia: clinicalpathologic correlation and review. AJNR Am J Neuroradiol 1986;7:61-72.

- 2 Yu YL, Moseley IF, Pullicino P, McDonald WI: The clinical picture of ectasia of the intracerebral arteries. J Neurol Neurosurg Psychiatry 1982;45:29-36.

3 Passero SG, Calchetti B, Bartalini S: Intracranial bleeding in patients with vertebrobasilar dolichoectasia. Stroke 2005;36:1421-1425.

$\longrightarrow 4$ Flemming KD, Wiebers DO, Brown RD Jr, Link MJ, Huston J 3rd, McClelland RL, Christianson TJ: The natural history of radiographically defined vertebrobasilar nonsaccular intracranial aneurysms. Cerebrovasc Dis 2005; 20:270-279.

$\checkmark 5$ Ubogu EE, Chase CM, Verrees MA, Metzger AK, Zaidat OO: Cervicomedullary junction compression caused by vertebral artery dolichoectasia and requiring surgical treatment. Case report. J Neurosurg 2002;96:140-143.

-6 Papapetropoulos S, Argyriou AA, Guevara A, Sengun C, Mitsi G, Singer C: Hemifacial spasm and pontine compression caused by a giant vertebrobasilar dolichoectasia. Cerebrovasc Dis 2009;27:413-414.

-7 Mangrum WI, Huston J 3rd, Link MJ, Wiebers DO, McClelland RL, Christianson TJ, Flemming KD: Enlarging vertebrobasilar nonsaccular intracranial aneurysms: frequen$\mathrm{cy}$, predictors, and clinical outcome of growth. J Neurosurg 2005;102:72-79.

8 Kwon HM, Kim JH, Lim JS, Park JH, Lee SH, Lee YS: Basilar artery dolichoectasia is associated with paramedian pontine infarction. Cerebrovasc Dis 2009;27:114-118.

$\checkmark 9$ Passero SG, Rossi S: Natural history of vertebrobasilar dolichoectasia. Neurology 2008; 70 : 66-72.

10 Wolfe T, Ubogu EE, Fernandes-Filho JA, Zaidat OO: Predictors of clinical outcome and mortality in vertebrobasilar dolichoectasia diagnosed by magnetic resonance angiography. J Stroke Cerebrovasc Dis 2008;17:388-393.

- 11 Lehoux S, Castier Y, Tedgui A: Molecular mechanisms of the vascular responses to haemodynamic forces. J Intern Med 2006;259: 381-392.

$\checkmark 12$ Nguyen KT, Clark CD, Chancellor TJ, Papavassiliou DV: Carotid geometry effects on blood flow and on risk for vascular disease. J Biomech 2008;41:11-19.

13 Nixon AM, Gunel M, Sumpio BE: The critical role of hemodynamics in the development of cerebral vascular disease. J Neurosurg 2010; 112:1240-1253.
14 Meng H, Wang Z, Hoi Y, Gao L, Metaxa E, Swartz DD, Kolega J: Complex hemodynamics at the apex of an arterial bifurcation induces vascular remodeling resembling cerebral aneurysm initiation. Stroke 2007;38: 1924-1931.

15 Nakatomi H, Segawa H, Kurata A, Shiokawa Y, Nagata K, Kamiyama H, Ueki K, Kirino T: Clinicopathological study of intracranial fusiform and dolichoectatic aneurysms: insight on the mechanism of growth. Stroke 2000;31: 896-900.

16 Boden-Albala B, Cammack S, Chong J, Wang C, Wright C, Rundek T, Elkind MS, Paik MC, Sacco RL: Diabetes, fasting glucose levels, and risk of ischemic stroke and vascular events: findings from the Northern Manhattan Study (NOMAS). Diabetes Care 2008;31:11321137.

17 Jiang H, Alperin N: A new automatic skeletonization algorithm for 3D vascular volumes. Conf Proc IEEE Eng Med Biol Soc 2004;2:1565-1568.

18 Gutierrez J, Bagci A, Gardener H, Rundek T, Ekind MSV, Alperin N, Sacco RL, Wright CB: Dolichoectasia diagnostic methods in a multiethnic, stroke-free cohort: results from the Northern Manhattan Study. J Neuroimaging 2013, E-pub ahead of print.

19 Willis T, Pordage S, Dring T, Harper C, Leigh J: Dr. Willis's Practice of Physick: Being the Whole Works of That Renowned and Famous Physician: Containing These Eleven Several Treatises, viz. London, Printed for T Dring, C Harper, and J Leigh, 1684.

20 van der Zwan A, Hillen B, Tulleken CA, Dujovny M: A quantitative investigation of the variability of the major cerebral arterial territories. Stroke 1993;24:1951-1959.

21 Hillen B: The variability of the circle of Willis: univariate and bivariate analysis. Acta Morphol Neerl Scand 1986;24:87-101.

22 Derdeyn CP, Videen TO, Yundt KD, Fritsch SM, Carpenter DA, Grubb RL, Powers WJ: Variability of cerebral blood volume and oxygen extraction: stages of cerebral haemodynamic impairment revisited. Brain 2002;125: 595-607.

23 Liebeskind DS, Sanossian N: How well do blood flow imaging and collaterals on angiography predict brain at risk? Neurology 2012; 79:S105-S109.

24 Padget D: The circle of Willis: its embryology and anatomy; in Dandy WE (ed): Intracranial Arterial Aneurysms. Ithaca, Comstock Publishing Company, Cornell University, 1944, pp 67-90.
25 Alpers BJ, Berry RG, Paddison RM: Anatomical studies of the circle of Willis in normal brain. AMA Arch Neurol Psychiatry 1959;81: 409-418.

26 Riggs HE, Rupp C: Variation in form of circle of Willis. The relation of the variations to collateral circulation: anatomic analysis. Arch Neurol 1963;8:8-14.

27 Leblanc GG, Golanov E, Awad IA, Young WL: Biology of vascular malformations of the brain. Stroke 2009;40:e694-e702.

28 Risau W: Mechanisms of angiogenesis. Nature 1997;386:671-674.

29 Van Overbeeke JJ, Hillen B, Tulleken CA: A comparative study of the circle of Willis in fetal and adult life. The configuration of the posterior bifurcation of the posterior communicating artery. J Anat 1991;176:45-54.

-30 Burger IM, Siclari F, Gregg L, Gailloud P: Bilateral segmental agenesis of the vertebrobasilar junction: developmental and angiographic anatomy. AJNR Am J Neuroradiol 2007;28: 2017-2022.

31 Kier LE: Section I. Fetal cerebral arteries: a phylogenetic and ontogenic study; in Newton TH, Potts DG (eds): Radiology of the Skull and Brain. Saint Louis, Mosby, 1971, pp 1089-1130.

-32 Krabbe-Hartkamp MJ, van der Grond J, de Leeuw FE, de Groot JC, Algra A, Hillen B, Breteler MM, Mali WP: Circle of Willis: morphologic variation on three-dimensional time-of-flight MR angiograms. Radiology 1998;207:103-111.

33 El-Barhoun EN, Gledhill SR, Pitman AG: Circle of Willis artery diameters on MR angiography: an Australian reference database. J Med Imaging Radiat Oncol 2009;53:248-260.

34 Riles TS, Eidelman EM, Litt AW, Pinto RS, Oldford F, Schwartzenberg GW: Comparison of magnetic resonance angiography, conventional angiography, and duplex scanning. Stroke 1992;23:341-346.

35 Patrux B, Laissy JP, Jouini S, Kawiecki W, Coty P, Thiebot J: Magnetic resonance angiography (MRA) of the circle of Willis: a prospective comparison with conventional angiography in 54 subjects. Neuroradiology 1994; 36:193-197.

36 Ford MD, Alperin N, Lee SH, Holdsworth DW, Steinman DA: Characterization of volumetric flow rate waveforms in the normal internal carotid and vertebral arteries. Physiol Meas 2005;26:477-488.

37 Cebral JR, Castro MA, Putman CM, Alperin $\mathrm{N}$ : Flow-area relationship in internal carotid and vertebral arteries. Physiol Meas 2008;29: 585-594. 